\title{
Synchrotron Phase Transition Crossing Using an RF Harmonic
}

\author{
J. E. Griffin \\ Fermi National Accelerator Laboratory \\ P.O. Box 500 \\ Batavia, Illinois 60510
}

March 1991 


\section{SYNCHROTRON PHASE TRANSITION CROSSING USING AN RF HARMONIC}

James E. Griffin

Fermilab 3-91

\section{INTRODUCTION}

This paper describes a new method of transition crossing in strong focussing proton or heavy ion synchrotrons. Such accelerators have the property that at some energy, frequently within the operating range of the machine, the rotation period of particles within the momentum acceptance range of the machine becomes independent of momentum. (1) For a small range of energy near this "transition energy" (or transition gamma, where $\gamma=\left(E / E_{0}\right)$ ), the phase focussing effect of the rf voltage wave on an ensemble of particles or "bunch" is temporarily lost. ${ }^{(2,3,4)}$ At energies below the transition energy the rf system provides a phase focussing force on each particle within each bunch in addition to the required accelerating voltage. This is accomplished by adjusting the phase of the rf wave so that the particles within each bunch oscillate about a point on the positive slope of the If wave at a "synchronous phase angle" $\varphi_{B}$. For a peak rf voltage $\mathrm{V}$ the rf accelerating voltage on each particle, averaged over a synchrotron phase oscillation, is $V \sin \left(\varphi_{s}\right)$. Above transition the phase stable point is shifted to a point of negative slope and the average accelerating voltage becomes $V \sin \left(\pi-\Phi_{\mathrm{B}}\right)$. For a time and energy range very near transition the rf system must continue to provide the required accelerating voltage to each particle, but longitudinal focussing is not necessary. The particles in each bunch, all of which have nearly identical rotation frequencies, cannot complete synchrotron phase oscillations, do not tend to debunch (ignoring, for the moment, internal space charge forces), and require no external phase focussing force. Any voltage other than the required accelerating voltage impressed upon particles within a bunch by the rf wave near transition 
may cause undesirable distortion of the phase space distribution and growth in the beam momentum spread. The "transition phase jump", the manner by which the rf system is programmed to make the required phase shift through the transition region while at the same time providing the required accelerating voltage, is the primary subject of the "transition crossing problem".

A substantial body of literature discussing the dynamics of particles during the transition period exists. ${ }^{(5-11)}$ Many of the analyses make the assumption that the rf amplitude remains constant at the required $\varphi_{s}$ until such time as a rather sudden phase jump is made. One problem with this procedure is that it is rarely clear exactly when the sudden phase jump should be made since in most cases particles with different momenta cross transition at different times. Other analyses examine the case in which the rf voltage is decreased as transition is approached so that "at transition" $\left(\varphi_{a}=\pi / 2\right)$ the amplitude is just the required accelerating voltage. This procedure is frequently called "duck-under". (12) The exact manner by which the voltage is lowered is somewhat arbitrary so the detailed dynamics of various duck-under schemes is not uniquely defined. The procedure described in this report has been motivated more by detailed examination of the results of large scale simulations of transition crossing using the longitudinal phase space simulation program ESME(13) than by analytical results, so that a detailed description of these analyses is not appropriate here. It will be useful, however, to enumerate some of the problems arising from transition crossing so that the manner by which this procedure circumvents these problems may be more clearly understood.

1. As transition is approached the effective if focussing force for a fixed $\varphi_{s}$ increases rapidly, the longitudinal phase space separatrices (bucket boundaries) expand in the momentum direction, and the bunches become very narrow in their phase or time extent. The bunch narrowing occurs at the expense of an increase in the bunch momentum spread, sometimes to the extent that the momentum spread exceeds the machine momentum aperture and beam loss occurs. 
2. Very near transition the particles enter a "non-adiabatic" period in which the particles are unable to complete a synchrotron phase oscillation prior to transition. During this period, if the rf phase is held at some $\varphi_{0}<\pi / 2$, particles arriving early receive insufficient accelerating voltage while late arriving particles are excessively accelerated. This causes the bunch phase space "ellipse" to tilt toward later time while further momentum growth occurs.

3. During this period the line charge density, or current density within the bunch, and the resulting internal space charge force is maximized. This force is capable of causing transverse (betatron) tune shift for some of the particles resulting in a change in the time at which these particles cross transition, (Umstätter effect). This further confuses the transition crossing time determination and may, in some cases, contribute to an increase in transverse emittance.

4. Immediately following transition, (probably individual times for each particle), the large space charge forces begin to act in reverse. ${ }^{(14,15)}$ (The effective mass of the particles in response to external forces becomes negative.) Because of the somewhat nonuniform initial distribution this causes a clumping in the line charge density of particles within the bunch or "negative mass instability". The clumping introduces very high frequency components in the line charge distribution which, in turn couple to resistive components of the beam pipe impedance in the GHz region. The longitudinal voltage resulting from this interaction couples back to the beam, causing further very high frequency spontaneous bunching, "microwave instability". The result of all of this is a further increase in the bunch momentum spread.

5. If, very near transition, the If phase is shifted to $\left(\pi-\varphi_{n}\right)$, then late arriving particles, still relatively immobile in the phase or time direction, receive insufficient accelerating voltage and are rapidly decelerated to momenta well below the synchronous momentum, while early arriving particles receive somewhat the opposite treatment. This effect is more serious for the late particles because, as they are being decelerated, the transition energy is 
moving simultaneously to lower energy, so these particles remain near transition for a long period, move toward later time (because they are below transition) and therefore experience an increasing decelerating voltage. In some circumstances some of these particles never cross transition, (or, transition never catches up to them as they are decelerated), so they are lost. In any case this effect causes a long low-momentum tail to develop. At some time after the end of the non-adiabatic period, when the rf system is again able to develop a reasonable bucket, this long tail of low momentum particles will extend across the separatrix so that some of the particles will not be captured in the bucket. The uncaptured particles will eventually be lost from the machine. Those that are recaptured will be badly matched to bucket and their mismatched motion within the bucket will contribute to "bunch shape oscillations" which are commonly observed following transition. Space charge distortion of the bunch shape will also contribute to these oscillations.

Some of the effects described above are shown in Fig.1 using the tracking program ESME. The evolution of a phase space contour of area $0.5 \mathrm{ev}-\mathrm{s}$ is shown at intervals over a period of $16 \mathrm{~ms}$ through transition with a "normal" rf phase jump occurring at $10 \mathrm{~ms}$. A vertical line of charge is included in the initial distribution and the area on one side of this boundary is cross-hatched so that the evolution of the distribution can be traced easily. The developing low momentum tail extending beyond the post-transition bucket boundary and the resulting bunch shape degradation are clearly shown. The example is based on the proposed Fermilab Main Injector ring with an acceleration rate of $150 \mathrm{GeV}$ per second. Transition gamma is 20.4. This simulation does not include any space charge effects.

It is evident that most of the ill effects of "normal" transition crossing result from early and late particles being subjected to incorrect $r f$ voltage. It should be possible then to shift the rf phase to $\pi / 2$ near the beginning of the non-adiabatic period and "duck under" transition. Unfortunately the particles are not quite rigidly stationary during this period. After the rf wave is shifted to $\pi / 2$, 


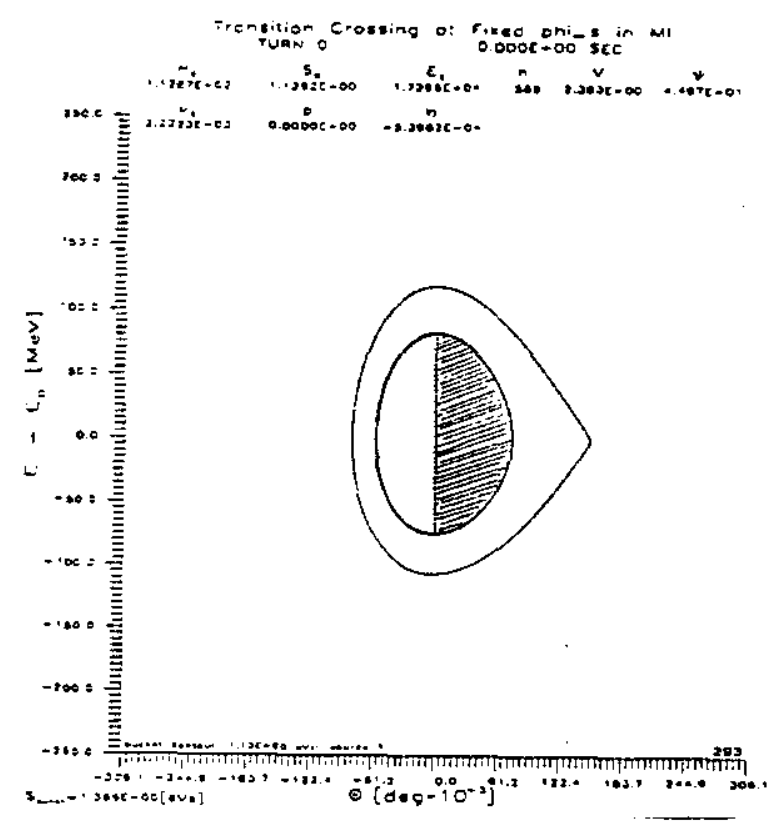

(a)

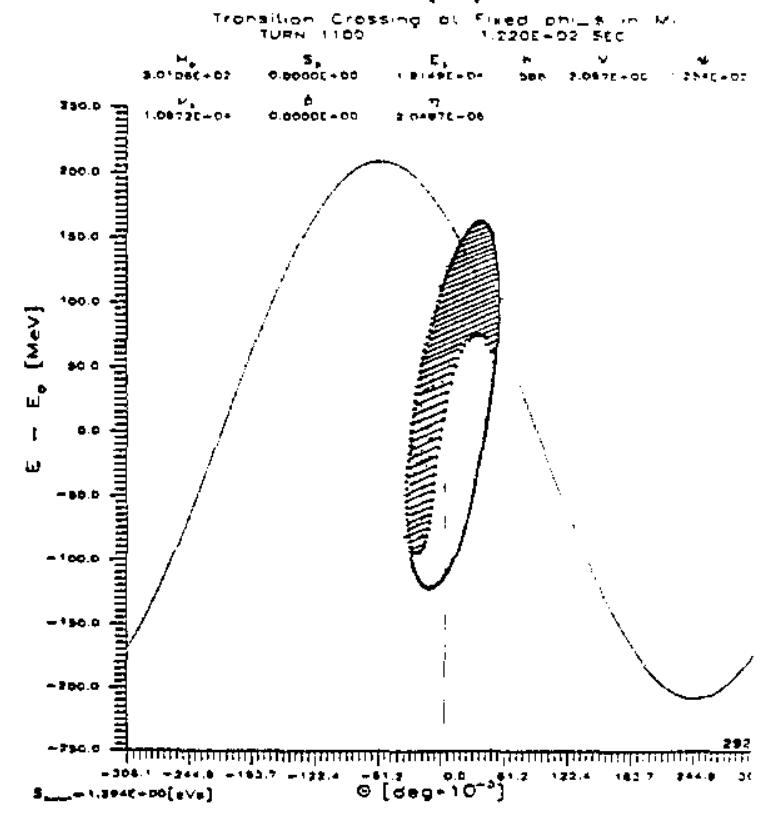

(c)

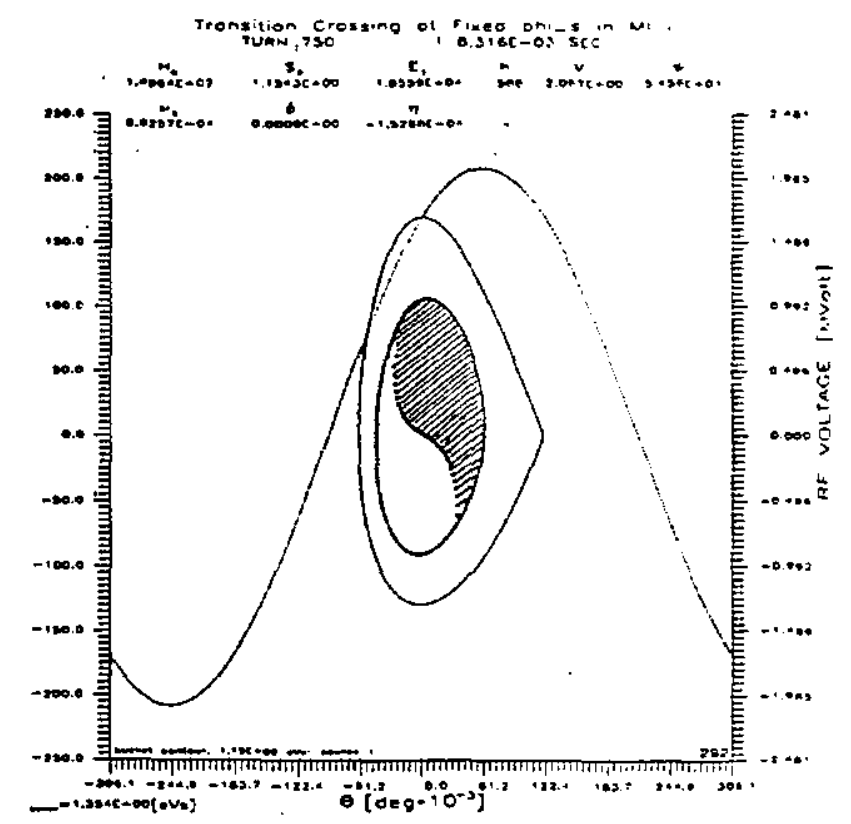

(b)

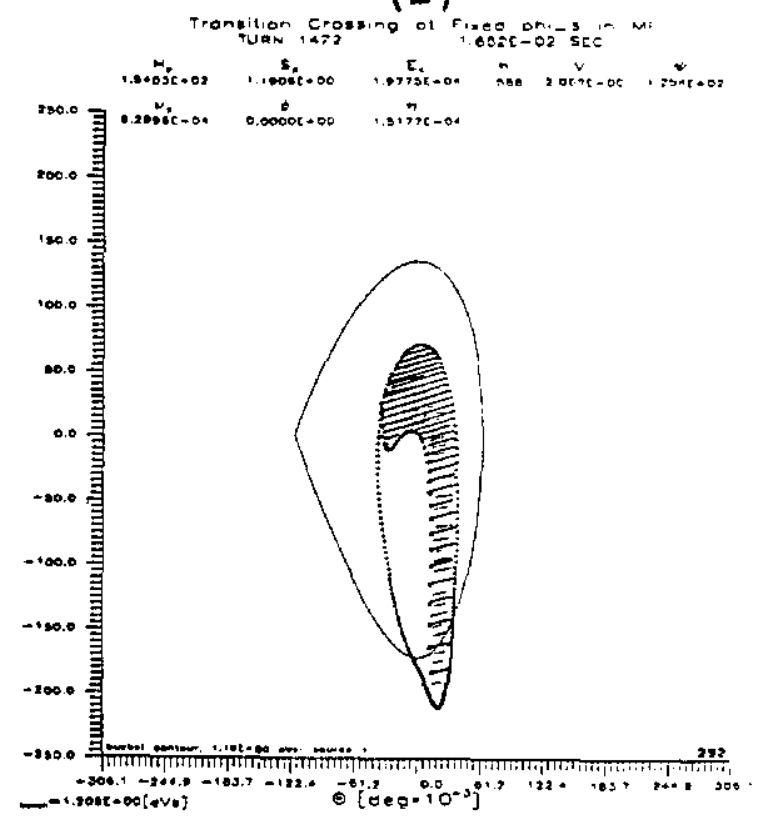

(d)

Figure 1. Normal transition crossing. The rf amplitude remains constant at $\varphi_{\text {. }}$ until transition at which time it is switched to $\pi-\varphi_{.}$. (a) $10 \mathrm{Ms}$ prior to transition. (b) Start of "non-adiabatic" period." (c) Transition. (d) 6 Ms after transition. 


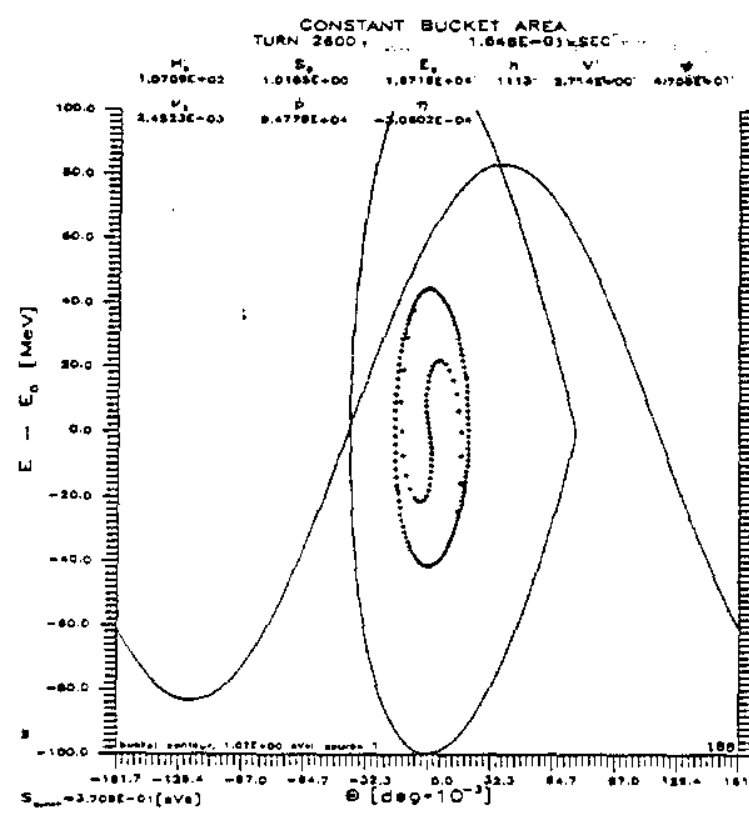

(a)

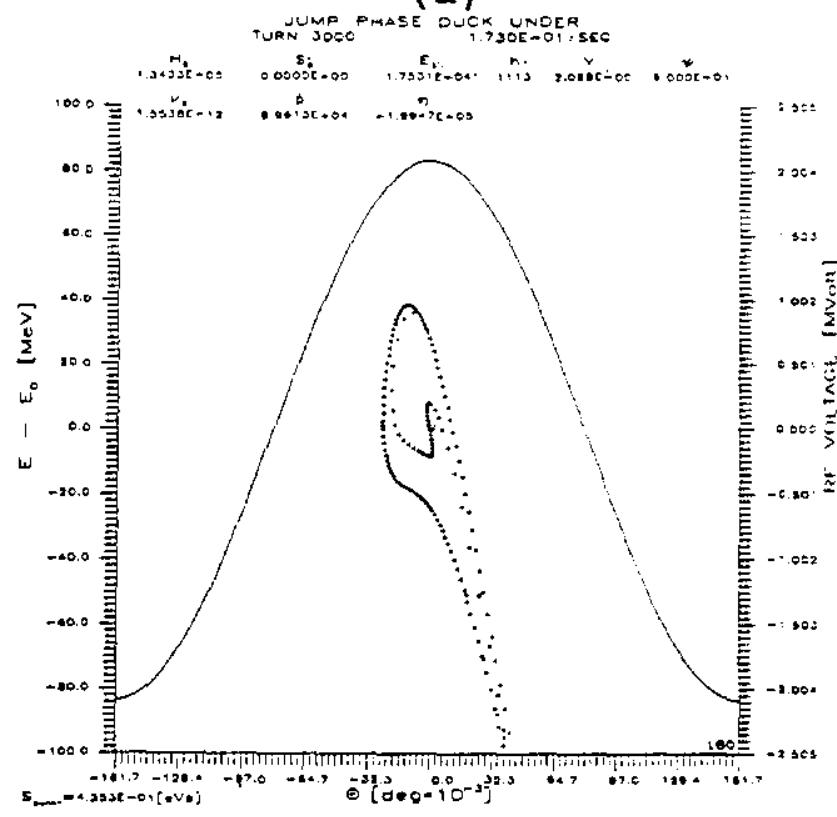

(c)

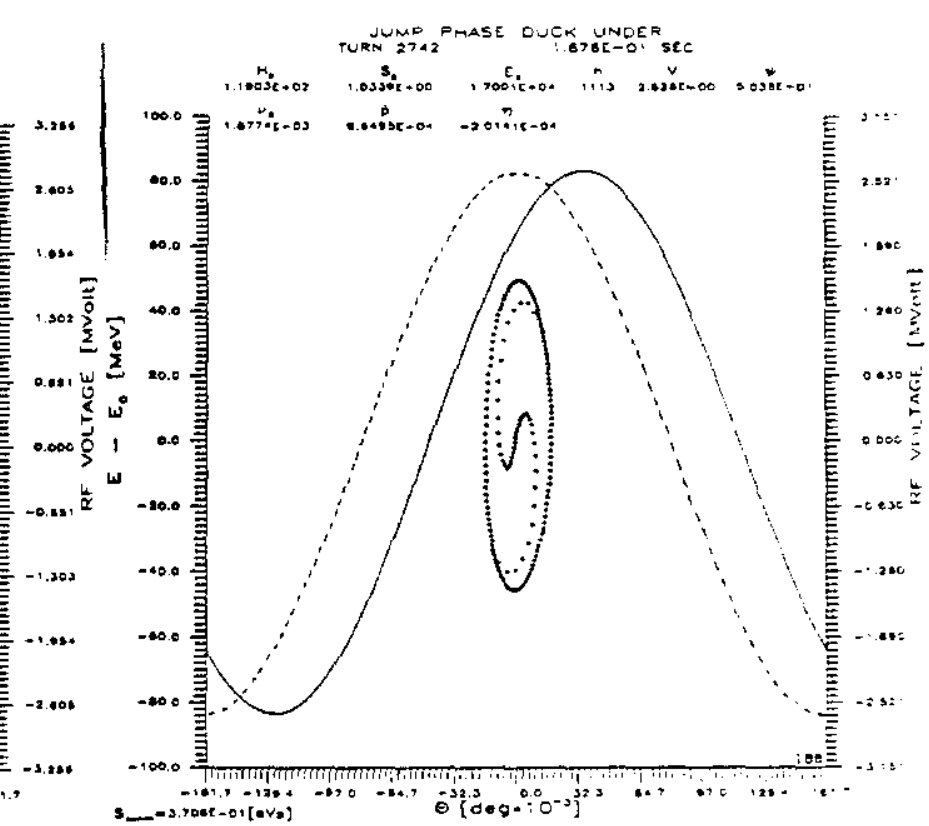

(b)

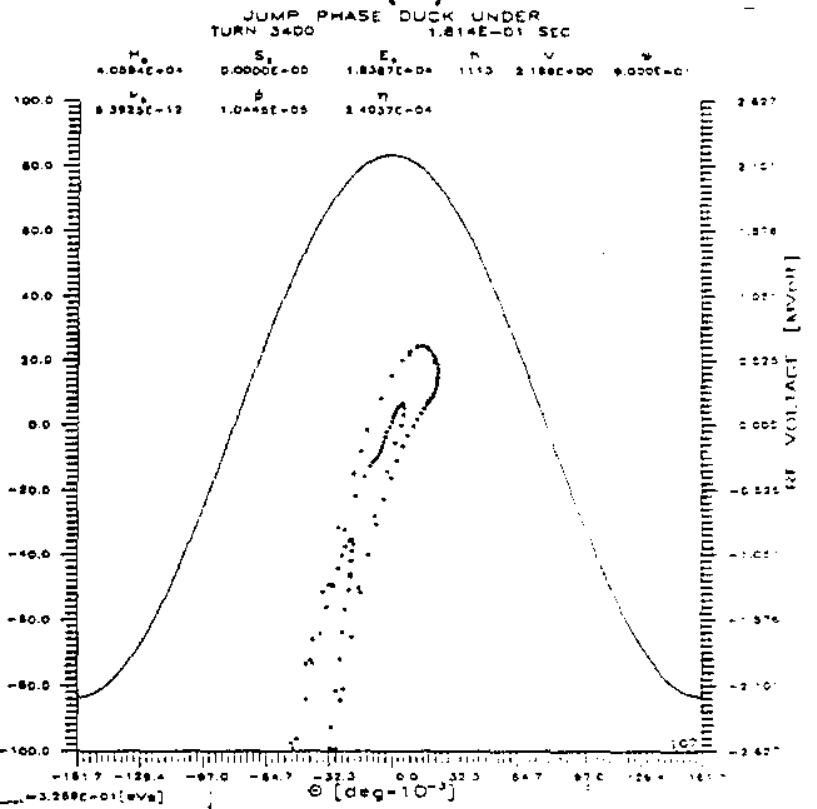

(d)

Figure 2. "Duck-under" transition crossing. At the start of the non-adiabatic period the $r$ phase is shifted to $\pi / 2$ radians and the amplitude reduced. (a) $6 \mathrm{~ms}$ prior to transition. (b) start of non-adiabatic period. (c) Very near transition. (d) $6 \mathrm{~ms}$ after transition. Here low momentum particles move to earlier time. 
higher momentum particles move to earlier time where they receive insufficient acceleration while lower momentum particles move to later time and suffer the same fate. The evolution of a contour similar to that shown in Fig. 1 is shown in Fig. 2 using duck-under phase changes. Again, particles away from the correct synchronous phase angle receive incorrect accelerating voltage on each turn for a substantial period of time so that large momentum changes result in serious distortion of the phase space distribution.

The evolutions shown in these two examples are extreme cases contrived to show clearly the effects described. Nevertheless beam loss, bunch shape oscillations, and longitudinal emittance growth following transition, all with properties very like those shown here, are commonly observed in many operating proton synchrotrons.

\section{TRANSITION CROSSING WITH AN RF HARMONIC}

At energies far from transition all particles in each bunch oscillate about the synchronous phase angle in such a way that each particle receives, on average, the correct accelerating voltage. A large part of the emittance growth which occurs during transition crossing results from the fact that this averaging cannot happen for a substantial period as the synchrotron phase oscillation frequency passes through zero. If, during this period, all of the particles in each bunch were accelerated by the same accelerating voltage on each turn the anomalous momentum spread could be eliminated. Of course, the accelerating voltage seen by each particle would have to be the correct voltage to keep the average beam position on the equilibrium orbit as dictated by the rate of rise of the magnetic guide field. This would require an $r f$ accelerating wave which is essentially constant over a substantial period of phase, or time, on either side of the bunch charge distribution. An rf voltage wave which meets this requirement can be generated by the addition of a second or third harmonic of proper amplitude and phase to the fundamental rf wave. combination of second and third harmonic could also be used but the gain may not be worth the added complexity.) Fig. 3 shows a sine wave with 27.5 \& second harmonic cosine wave added. The amplitude is 


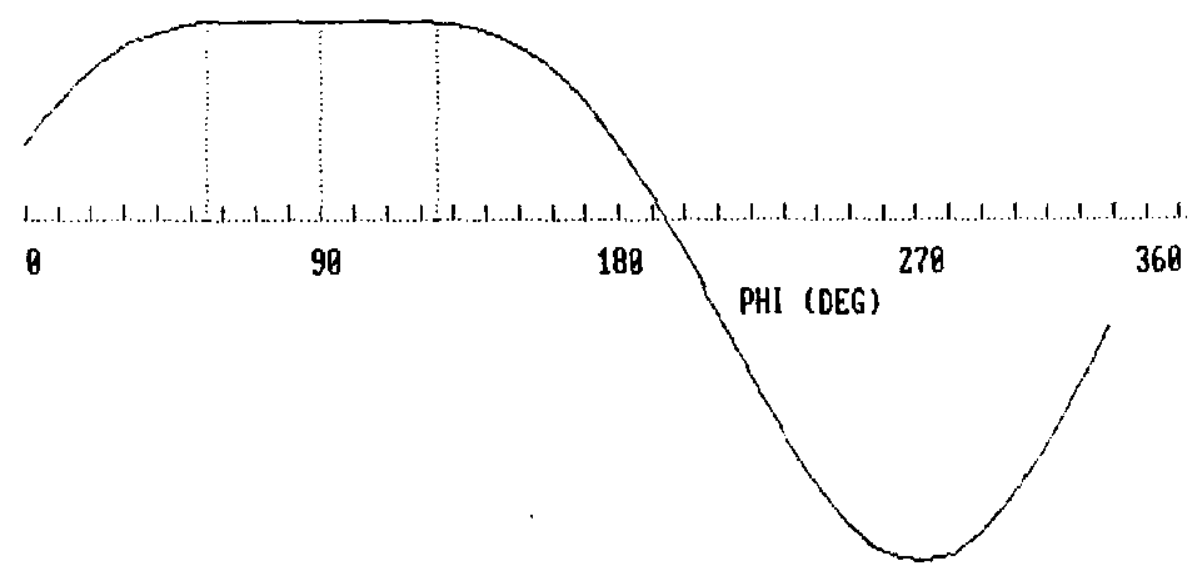

Figure 3. $v(\omega t)=\sin (\omega t)+(0.275) \cos (2 \omega t)$

constant within \pm .25 per cent over a range of \pm 35 degrees.

In Fig.4 the transition crossing procedure is outlined. In this example the synchronous phase angle prior to the non-adiabatic period is sixty degrees and the bucket to bunch area ratio is about three. The bucket extends over a phase range of ninety-one degrees and the total length of the contour representing the bunch is about forty-four degrees. At some time prior to the beginning of the non-adiabatic period (usually a few milliseconds before "transition") the rf phase is shifted to $\pi / 2$ radians and the second harmonic is added as shown in part $b$ of the figure. The bunch immediately begins to shear, with higher momentum particles moving to earlier time and lower momentum particles moving to later time. At transition time the bunch has sheared to the condition shown in part $c$ with no change in relative momentum. At this time the line charge distribution (projection of the charge on the time, or phase, axis) has broadened as shown, with a corresponding decrease in peak current and space charge force. Following transition the direction of motion of off-momentum particles is reversed. As time progresses past the end of the non-adiabatic period the bunch phase space distribution resumes its original upright 


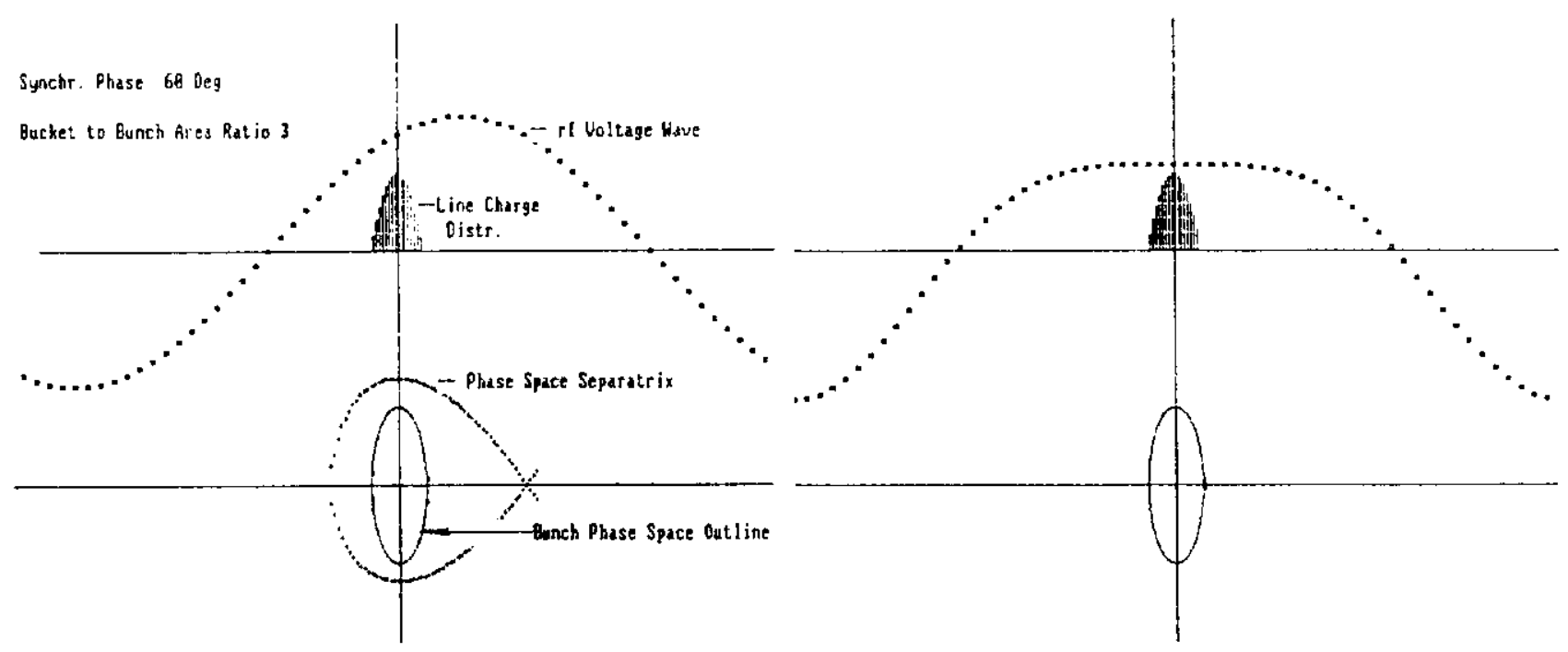

(a)

(b)

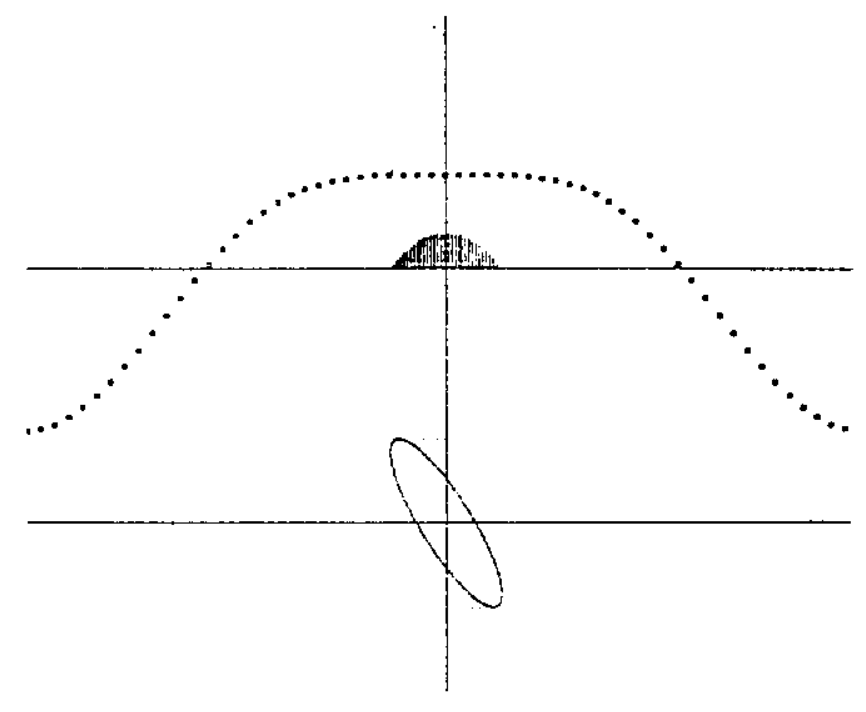

(c)

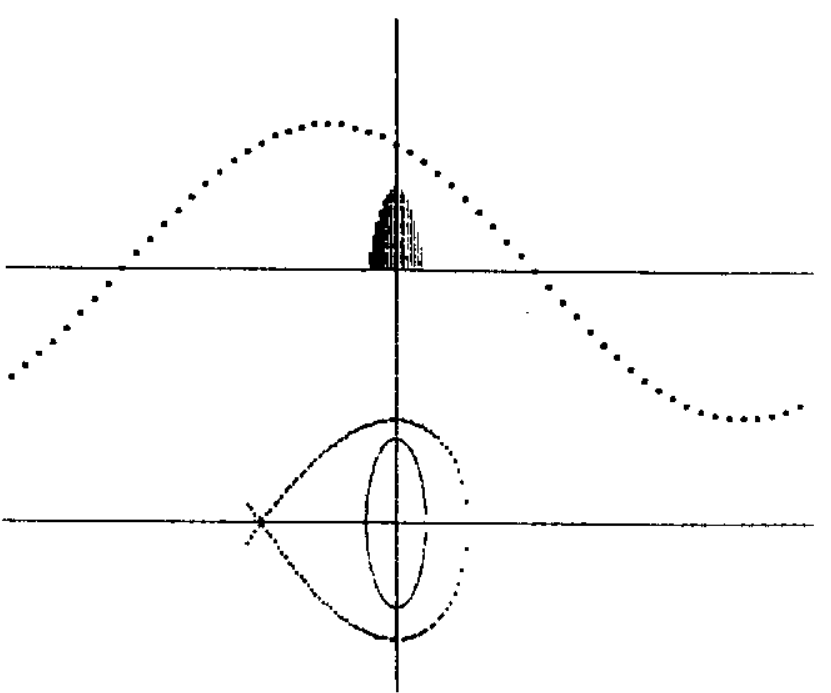

(d)

Figure 4. (a) Phase space contour, moving bucket separatrix, and line charge distribution with respect to fundamental rf voltage wave prior to transition with synchronous phase angle 60 degrees. (b) Phase space outline and line charge distribution at the same time, but with rf voltage wave shifted to ninety degrees and second harmonic added. (c) Same beam representations a few milliseconds later, at transition time. (d) A few milliseconds after transition. The phase space contour has become erect again. The rf wave has been shifted to a post transition accelerating phase and an accelerating bucket is shown. 
configuration. At this time the second harmonic component is removed and the $r f$ wave is shifted to the correct phase angle and amplitude for longitudinal focussing and acceleration following transition.

This procedure does not neutralize internal forces resulting from space charge. However the sign of the space charge force changes from defocussing to focussing at transition. Except for the possibility of negative mass and microwave instability following transition, the bunch lengthening effect of space charge forces during the non-adiabatic period should cancel to first order. The total bunch length at transition may, however, be increased somewhat beyond that which results from shearing alone prior to transition.

In some cases it may be possible the use the third harmonic rather than the second although it provides only about \pm 27 degrees of comparable constant amplitude. Fig.5 shows an expanded comparison of both harmonics. Use of the third harmonic would be clearly less expensive since only 13 per cent of the fundamental amplitude is required so fewer and physically smaller rf cavities would be required.

$\operatorname{SIN}(\mathrm{PHI})+.1295 \operatorname{SIN}(3 * \mathrm{PHI}) \circ 00$

$\sin (P H 1)+.275 * \operatorname{Cos}(2 * \mathrm{PHI})-$

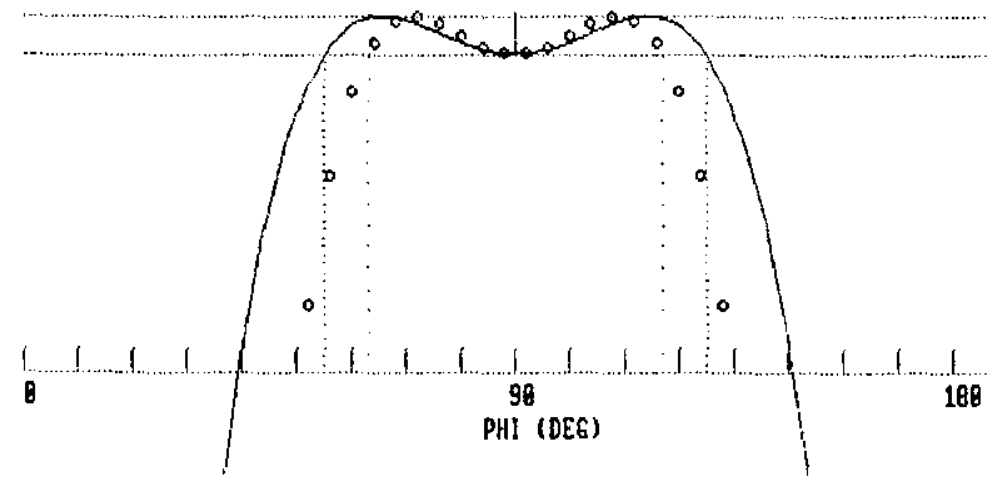

Figure 5. Expanded comparison of constant voltage regions available using second or third harmonic. 


\section{NON-ADIABATIC and NON-LINEAR PERIOD AROUND TRANSITION}

The total phase space available for bunch manipulation near transition with rf focussing removed is delimited by the safe momentum aperture and by the time or phase range of constant voltage created by whichever rf harmonic is chosen. The extent of bunch lengthening during the non-adiabatic period is affected by the initial conditions of bunch momentum and phase spread and by the duration of the non-adiabatic period. These conditions are set by the bunch longitudinal emittance contour (that containing 958 of the charge in this development), and by the rf bucket to bunch area ratio established by the rf voltage and synchronous phase angle at the beginning of the non-adiabatic time. Expressions for the non-adiabatic time have been presented in several places. ${ }^{(5,14)}$ Here we express the non-adiabatic time $t_{1}$ (before and after transition) in terms of the machine rotation period $T_{\circ}$.

$$
t_{1}= \pm T_{o}\left[\frac{E_{o} F_{o} \gamma_{t}^{4}}{4 \pi h \dot{\gamma} V \mid \cos \left(\varphi_{s}\right)}\right]^{1 / 3} .
$$

where

$$
\begin{aligned}
& E_{0}=\text { particle rest mass energy, } \\
& F_{0}=1 / T_{\circ} \text { rotation frequency of reference particle, } \\
& \gamma_{t}=\text { transition gamma of reference particle, } \\
& \dot{\gamma}=\text { d } / \text { dt, acceleration rate, } \\
& h=\text { fundamental harmonic number, } \\
& V=\text { peak rf voltage. }
\end{aligned}
$$

For the Fermilab Main Ring, $\left(\gamma_{t}=18.75, h=1113\right)$, operating at acceleration rate $85 \mathrm{GeV} / \mathrm{s}$ through transition, $t_{1}$ is approx. 170 turns, or $3.5 \mathrm{~ms}$ if $\varphi_{\mathrm{a}}$ is $\pi / 3$ and $V_{\mathrm{rf}}$ is $2 \mathrm{MV}$ at the beginning of the non-adiabatic period. In most accelerating circumstances $t_{1}$ is a few milliseconds.

Approximate adiabaticity exists at times removed from transition by $\pm t_{1}$. But transition is a local effect, usually occurring at different times for different increments of momenta within the aperture. In order to ensure reasonable adiabaticity for all particles within a distribution it is necessary to determine the time period 
required for the entire distribution to cross transition (the "non-linear" period, here labelled $t_{2}$ ) and add it to the non-adiabatic time, $t_{1}$. The following derivation of $t_{2}$ follows that of $\mathrm{K}$. Johnsen, but uses a different definition for $\alpha_{1} \cdot{ }^{(16)}$

The difference in orbit length, $\Delta L=L-L$ 。 between a particle at momentum $p=p_{0}+\Delta p$ and a reference particle is expressed

$$
\frac{\Delta L}{L_{0}}=\alpha_{0}\left(\frac{\Delta p}{p_{0}}\right)+\alpha_{1}\left(\frac{\Delta p}{p_{0}}\right)^{2}
$$

where $\alpha_{0}=\gamma_{t}^{-2}$ is the "momentum compaction factor"(1), $\alpha_{1}$ is a chromatic error term, $\left(\approx .0023\right.$ in the Fermilab Main Ring, $\left.{ }^{(17)}\right)$ and $\gamma_{t}$ is the transition $\gamma$ for the reference orbit. Transition $\gamma$ for off-momentum particles in an incremental region near $p$ is defined

$$
\frac{1}{\gamma_{t}^{2}(\delta)}=\frac{p}{L} \frac{d L}{d p}=\alpha_{0}+\left(\alpha_{0}+2 \alpha_{1}-\alpha_{0}^{2}\right) \delta
$$

where $\delta=\Delta p / p_{0}$. To first order in $\delta$

$$
\gamma_{t}(\delta)=\gamma_{t}\left\{1-\left(\frac{1}{2}+\frac{\alpha_{1}}{\alpha_{0}}\right) \delta\right\}
$$

At any specific time off-momentum particles can be related to particles on the reference orbit by;

$$
\begin{gathered}
\gamma(\delta)=\gamma_{0}+\Delta \gamma=\gamma_{0}\left(1+\frac{\Delta \gamma}{\gamma_{0}}\right)=\gamma_{0}\left(1+\beta^{2} \delta\right), \\
\text { or } \quad \gamma_{0} \approx \gamma(\delta)\left(1-\beta^{2} \delta\right) .
\end{gathered}
$$


By combining Eqns. (4) and (5) $\gamma_{0}$ at the time when off-momentum particles are at transition, (here labelled $\gamma_{o t}$ ), can be expressed to first order in $\delta$,

$$
\begin{gathered}
\gamma_{o t}=\gamma_{t}\left[1-\left(\beta^{2}+1 / 2+\alpha_{1} / \alpha_{0}\right) \delta\right] \\
\text { or } \quad\left(\gamma_{O t}-\gamma_{t}\right) / \gamma_{t}=-\left(\beta^{2}+1 / 2+\alpha_{1} / \alpha_{0}\right) \delta .
\end{gathered}
$$

If $\dot{\gamma}$ is assumed to be constant over a time period $\pm t$ near reference orbit transition time $t_{t}, \gamma_{0}$ can be approximated by

$$
\gamma_{0}=\gamma_{t}+\dot{\gamma} t=\gamma_{t}\left(1+\dot{\gamma} t / \gamma_{t}\right) \quad-t \leq 0 \leq t
$$

By equating $\gamma_{0}$ in Eq. (7) to $\gamma_{\text {ot }}$ and substituting in Eq. (6), the time increment between the time off momentum particles reach transition and transition time for reference orbit particles can be expressed;

$$
t_{2}= \pm \frac{\gamma_{t}\left(\beta^{2}+1 / 2+\alpha_{1} / \alpha_{0}\right) \delta}{\dot{\gamma}}
$$

For the Fermilab Main Ring with $\dot{\gamma}=89$ at transition and $\delta= \pm 3 \times 10^{-3}$, $t_{2}$ is about $\pm 1.5 \mathrm{~ms}$.

The minimum time period required for non-focussing transition crossing now becomes $t_{n}= \pm\left(t_{1}+t_{2}\right)$, or about $\pm 5 \mathrm{~ms}$ for the example at hand. 


\section{SHEAR EXTENT DURING NON-FOCUSSING PERIOD}

It remains to establish a relationship between a typical $t_{n}$, the non-focussing time span available on the rf wave, and the bunch momentum spread at the beginning of the non-focussing period.

The angular velocity of a particle with momentum $p=p_{0}+\Delta p$ is expressed $\Omega=\Omega_{0}+\Delta \Omega$. The phase shift of such a particle with respect to a synchronous particle in a time interval $t$ is

$$
\Delta \theta=\int_{0}^{t} \Delta \mathbf{Q} d t
$$

The angular velocity of a particle on an orbit of length $L$ is;

$$
\Omega=\frac{2 \pi \beta C}{L}
$$

Using Eq. (2), the differential angular velocity $\Delta \Omega$ of off-momentum particles, referred to the reference orbit, is,

$$
\begin{aligned}
\Lambda \Omega & =2 \pi c\left[\frac{\beta}{L}-\frac{\beta_{0}}{L_{0}}\right]=\frac{2 \pi c \beta}{L_{0}}\left[\frac{(1+\Delta \beta / \beta)}{(1+\Delta L / L)}-1\right] \\
& =\Omega_{\circ}\left[\frac{\Delta \beta}{\beta_{0}}-\frac{\Delta L}{L_{0}}+\text { h.o.t. }\right] \approx \Omega_{0}\left[\frac{1}{\gamma_{0}^{2}}-\alpha_{0}-\alpha_{1} \delta\right] \delta .
\end{aligned}
$$

Inserting Eq.(7) into Eq.(11) and expanding,

$$
\Delta \Omega=-\Omega_{0}\left[\frac{2 \alpha_{0} \dot{\gamma} t}{\gamma_{t}}+\alpha_{1} \delta+h .0 . t .\right] \delta
$$

Using $\Delta \Omega$ from Eq.(12), the integral (9) can be evaluated over a time interval $-t_{n}$, the start of the non-focussing period, to $t$, giving the relative phase shift of an off-momentum particle with respect to a reference particle at any time $t$ during the non-focussing period. (To get rf radians $\Delta \Theta$ must be multiplied by the harmonic number; $\Delta \phi=h \Delta \Theta$. ) 


$$
\Delta \varphi(t)=h \Omega_{0} \delta\left[\frac{\alpha_{0} \dot{\gamma}\left(t_{n}^{2}-t^{2}\right)}{\gamma_{t}}-\alpha_{1} \delta\left(t_{n}+t\right)\right]
$$

By setting $t=0$ in (13), (transition time for a reference particle), the maximum shear, $\Delta \varphi$, of particles with momentum spread $\delta$ is obtained. For higher momentum particles, (positive $\delta$ ), the $\alpha_{1}$ term subtracts from the $\alpha_{0}$ term whereas for negative $\delta$ the two terms add. Positive phase shift implies relative movement to earlier time. If $\alpha_{1}$ is positive, particles with positive relative momentum reach transition slightly sooner than those with negative relative momentum and reverse their shear direction sooner. The maximum shear excursion is therefore smaller for positive $\delta$ particles.

At $t=+t_{n}$, the end of the non-adiabatic period, the $\alpha_{\circ}$ term in Eq. (13) drops out, leaving a total phase shift $\Delta \varphi=-2 h \Omega_{0} \alpha_{1} t_{n} \delta^{2}$. Unless $\alpha_{1}=0$, off momentum particles of both signs are shifted to later time; i.e. a distribution of particles extending over $\pm \delta$, all of which start at $\phi_{a}$, will end up still distributed over $\pm \delta$ but on a parabola opening toward later time.

These effects are illustrated in Fig.6. The initial condition shown in Fig.6a is a bunch outline of area $.18 \mathrm{eV}-\mathrm{s}$ matched to an accelerating bucket of area $.66 \mathrm{eV}-\mathrm{s}$. An additional line charge is shown spanning the momentum distribution at $\varphi_{s}$. The initial if voltage is $1.9 \mathrm{MV}$ and $\phi \mathrm{s}$ is $60 \mathrm{deg}$. The acceleration rate $\dot{\gamma}$ is assumed to be 85 throughout the transition period. Other parameters in the example are those of the Fermilab Main Ring. The non-adiabatic period for these conditions is approx. $\pm 5 \mathrm{~ms}$. Part b of the figure shows the sheared distribution at transition. The positive peak of the distribution has moved $15.7 \mathrm{deg}$. earlier while the negative peak has moved $21.7 \mathrm{deg}$. later. The $\alpha_{1}$ contribution at this point has been to move both peaks 3 deg. later. In part $c$, the distribution is shown 5-ms after transition. The shearing effect of the $\alpha_{0}$ term has disappeared and both peaks are moved $6 \mathrm{deg}$. later by the $\alpha_{1}$ term. 

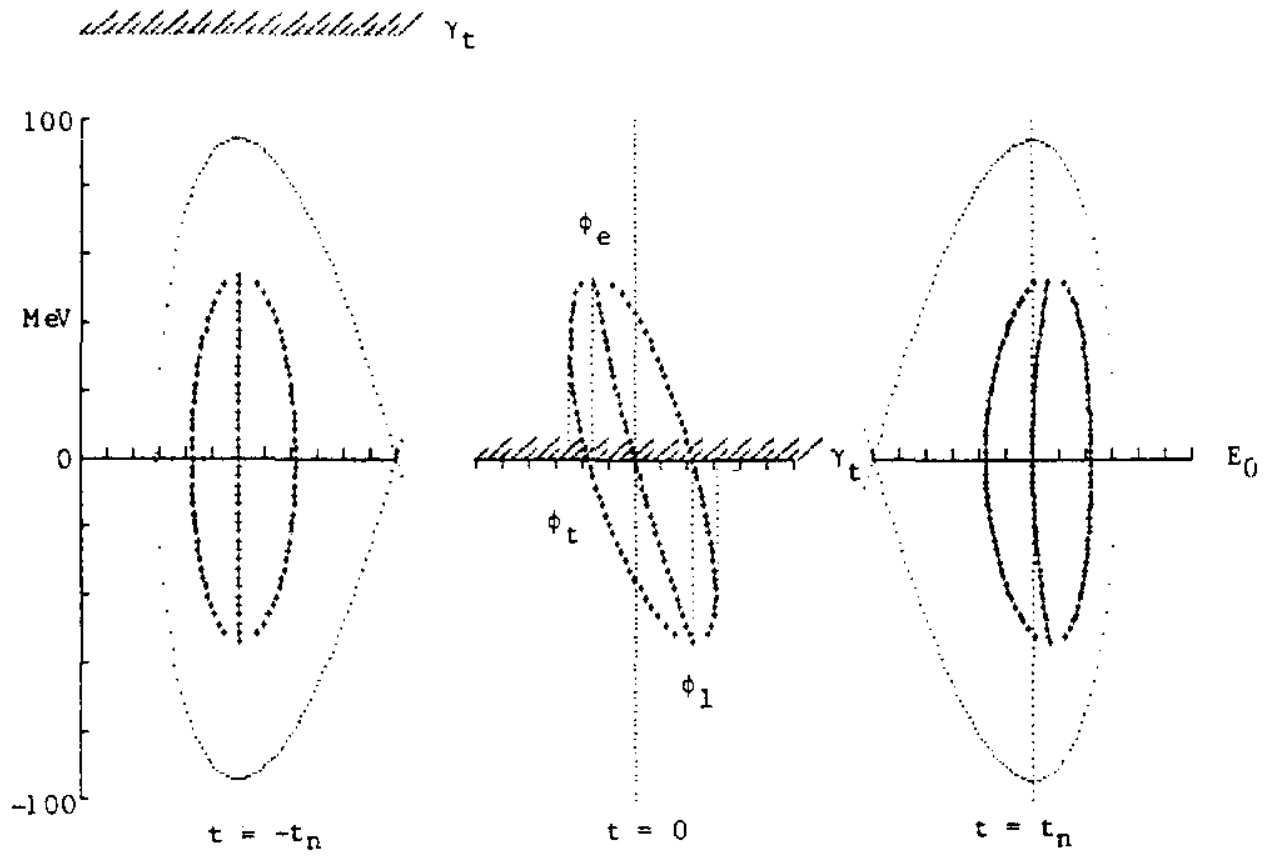

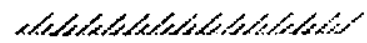

Figure 6. a) $.18 \mathrm{eV}-\mathrm{s}$ bunch outline matched to an accelerating bucket of area $.66 \mathrm{eV}-\mathrm{s}, 5 \mathrm{~ms}$ prior to transition. b) Bunch outline sheared in non-focussing rf regime at transition. c) The same bunch outline $5 \mathrm{~ms}$ after transition. The original outline has been distorted by chromatic $\left(\alpha_{1}\right)$ effect during transition crossing. An accelerating bucket has been outlined centered at $\pi-\phi_{3}$.

Cross-hatched regions in each part of the figure represent the relative position of $\gamma_{t}$ with respect to the synchronous energy at each time. Initially all particles are below transition. In part b the synchronous energy is equal to the transition energy and at this time all off-momentum particles are moving toward later $t$ ime. The particles in the distribution move progressively through transition, changing their direction of relative motion as they do, until finally the entire distribution is above transition, as in part $c$. The time period $2 t_{2}$ required for all the particles to pass through transition is affected by the sign and magnitude of $\alpha_{1}, \mathrm{Eq} \cdot(8)$.

During the entire transition crossing period the bunch centroid migrates in phase (time) with respect to the phase of the reference rf wave. This drift toward later time dictates that a slightly greater effective range of constant If accelerating voltage can be established if the phase of the fundamental and concomitant second (or third) 
harmonic wave is delayed a few degrees beyond ninety degrees during the non-focussing period.

In Fig. 6c, the post-transition accelerating bucket has been plotted with its stable fixed point at $\pi-\phi$. with respect to the phase of the starting if wave. This is evidently not the correct phase for the accelerating bucket, which should also be moved a few degrees toward later time to achieve an optimum match.

\section{APPROPRIATE CHOICE FOR $\alpha_{1}$}

As alluded to earlier, the potential for longitudinal blow-up and beam loss during "normal" transition crossing exists if the nonlinear time period is not substantially shorter than the non-adiabatic time. Adjustment of $\alpha_{1}$ to minimize the nonlinear time period by appropriate choice of sextupole field correction in the accelerator magnet lattice has been examined. ${ }^{(18)}$ However in the transition crossing procedure described here the presence of a particular positive value for $\alpha_{1}$ can be seen to be potentially beneficial. The equilibrium particle distribution within an accelerating bucket prior to transition is observed to be asymmetric with a sight bulge extending toward later time. The effect of positive $\alpha_{1}$ during the non-focussing transition period is to force all off-momentum particles toward later time and, in effect, to reverse the asymmetry in the distribution, causing it to create the possibility for better matching to the accelerating bucket following transition. This can be seen clearly in Fig. 6c. It is, however, possible that the $\alpha_{1}$ induced distortion, coupled with the chosen nonfocussing period, can result in a final distribution which is crescent shaped. This is an excessive and undesirable result.

If the distribution has not been allowed to become crescent shaped, both the maximum phase spread and the maximum momentum spread should be very similar to those of the initial distribution so a reasonable low intensity approximation to the correct bucket area and height should be achieved by applying the same rf voltage as existed at the beginning of the non-focussing period. (This is true only if the acceleration rate is not changing during the transition crossing period.)

In addition to the chromatic effects described here, the contour 
shape and phase space density of particles within the bunch following transition will be affected by space charge forces during the nonfocussing period. Application of accelerating rf of the correct phase and amplitude following transition crossing will, in general, be an experimental process, the success of which may be judged by minimization of bunch shape oscillation following transition.

\section{OPTIMIZATION OF INITIAL CONDITIONS}

If, in Eq. (13), $\delta$ represents the maximum momentum spread of the bunch, then the $\Delta \varphi$ obtained will also refer to particles with maximum momentum spread. As can be seen in Fig. 6b, this is not the total phase extent of the distribution at transition. The initial distribution may be conveniently represented by two half-ellipses with the same maximum energy spread but with different phase spreads $\varphi_{0}$ and $\boldsymbol{\varphi}_{1}$. Shearing of the two halves can be treated separately. If the effect of the $\alpha_{1}$ term is neglected in this treatment, the two sides of the distribution will shear into rotated and stretched half-ellipses with the initial phase spread and the shear phase shift adding in quadruture to give the maximum phase extent $\varphi_{t}$.

$$
\varphi_{t}=\left[\varphi_{\theta}^{2}+\varphi_{s}^{2}\right]^{1 / 2}+\left[\varphi_{I}^{2}+\varphi_{s}^{2}\right]^{1 / 2}
$$

The phase variables appearing in either of the terms of Eq.(14) are clearly not independent and they can be manipulated to optimize use of the available phase space during the non-adiabatic period. Consider the bunch phase space contour to be a symmetric ellipse with half phase spread $\varphi$ and area $S$, where $\varphi$ is the average of $\varphi_{0}+\varphi_{1}$. The maximum shearing phase $\varphi_{\mathrm{a}}$ is proportional to the momentum spread $\delta$ which is inversely proportional to the contour phase spread $2 \varphi$. $\mathrm{Eq} \cdot(14)$ can be rewritten approximately;

$$
\varphi_{t}=2\left[\varphi^{2}+\frac{M^{2}}{\varphi^{2}}\right]^{1 / 2} \quad \text { where } \quad M=\frac{4 \pi S h^{2} F_{o}^{2} t_{n}^{2} \dot{\gamma} \alpha_{o}^{2}}{\beta^{2} E_{o}}
$$


In Eq. (15) the minimum value of $\varphi_{t}$ occurs when $\varphi=\sqrt{M}$. If $\varphi$ is evaluated using the parameters of the above example the result is $\varphi= \pm .333 \mathrm{rad}( \pm 19 \mathrm{deg}$.$) which is very close to the initial condition$ used in the example. This implies an energy spread of the initial contour $\pm 56 \mathrm{MeV}$, or $\Delta p / p \approx \pm 0.338$. In general the optimum bunch phase spread arrived at using Eq.(15) is narrower and the corresponding momentum spread larger than acceptable, i.e. approaching or exceeding the machine momentum aperture. The implication is that optimum nonfocussing transition crossing is achieved (i.e. the largest longitudinal emittance can be accommodated), when the largest allowable momentum spread is established initially, or, when as much of the available phase space as possible is used. The rf voltage and synchronous phase angle should be adjusted to give the optimum bunch aspect ratio imnediately before switching to the non-focussing condition.

Optimization of the momentum spread prior to starting nonfocussing transition crossing allows the maximum crossing time to be used. This is important because of the $\alpha_{1}$ dependent momentum asymmetry in the bucket shape (and in the bunch momentum spread) which may arise as transition is approached. Higher momentum particles are closer to transition, move in phase more slowly, and consequently reach larger momentum spread than particles which are below the reference momentum. By starting the non-focussing period as early as possible this asymmetry may be minimized.

The initial phase spread for contours partially filling moving buckets may be obtained using the procedure of Cole and Morton. ${ }^{(19)}$ For the example of Fig. 6, $\varphi_{0}$ and $\varphi_{1}$ are $17.7 \mathrm{deg}$. and $21.8 \mathrm{deg}$. The shear angle $\varphi_{\text {. }}$ resulting from the $\alpha_{\circ}$ term only is $18.7 \mathrm{deg}$. resulting in a total phase spread from Eq.(13) of $54.4 \mathrm{deg}$. The total phase spread shown in Fig.6b is approx. $56 \mathrm{deg}$. ( $\pm 28 \mathrm{deg} \cdot$ ), well within the $\pm 35 \mathrm{deg}$. of non-focussing if voltage provided by the second harmonic component.

Space charge and asymmetry effects on non-focussing transition crossing can be evaluated using ESME simulations. 
ESME SIMULATIONS

Many ESME simulations of non-focussing transition crossing have been run, all with substantial success. Here, two such simulations are presented showing two very different situations.

The first example represents the Fermilab Main Ring with $3 \times 10^{10}$ protons per bunch within a (95\%) longitudinal emittance contour of area $0.2 \mathrm{eV}-\mathrm{s}$. The ring impedance $\mathrm{z} / \mathrm{N}$ is assumed to be 9 Ohms with a broad band resonant frequency $1.29 \mathrm{GHz}$. Transition $\gamma$ is 18.75 and $\alpha_{1}$ is assumed to be 0.0023 . Transition occurs within a parabolic region of momentum increase and $\dot{\gamma}$ at transition is 88.7. The non-focussing time $t_{n}$ is $\pm 6 \mathrm{~ms}$. Using these parameters the optimum initial bunch length is found from Eq.15 to be $40.8 \mathrm{deg}$. This implies an initial energy spread $\pm 56 \mathrm{MeV}(\delta= \pm 0.00328)$, assuming that the energy spread is symmetric about the reference energy.

In Fig.7 the evolution of a $0.2 \mathrm{ev}-\mathrm{s}$ contour with the above initial conditions is shown over a $12 \mathrm{~ms}$ period centered on transition. The contour shapes are those predicted by Eq.13 and no space charge effects are introduced. The vertical dotted lines in the figure represent the \pm 35 degree non-focussing range available. In part $b$ of the figure the contour is at its maximum shear point. Particles shearing to earlier time have not reached the limit of constant rf voltage whereas those shearing to later time have passed beyond the boundary. A small phase shift of the entire rf waveform toward later time could optimize the use of the non-focussing region of the wave.

In Fig. 8 an ESME generated distribution is shown occupying the initial contour. This distribution was generated by starting with a $0.2 \mathrm{eV}-\mathrm{s}$ (958) distribution matched to an accelerating bucket at $\gamma=15$ and tracking normal acceleration until 6 ms prior to transition, at which point the rf voltage was programmed to reach $2.32 \mathrm{MV}$ with $\Phi_{8}=47 \mathrm{deg}$. The phase spread of the distribution in part a of the figure is about 42 degrees, quite close to the optimum value. The energy spread is slightly asymmetric, $+57,-55 \mathrm{MeV}$. Part b shows the sheared distribution very near transition. The rf wave has been delayed 6 degrees so that the constant voltage region matches the sheared 


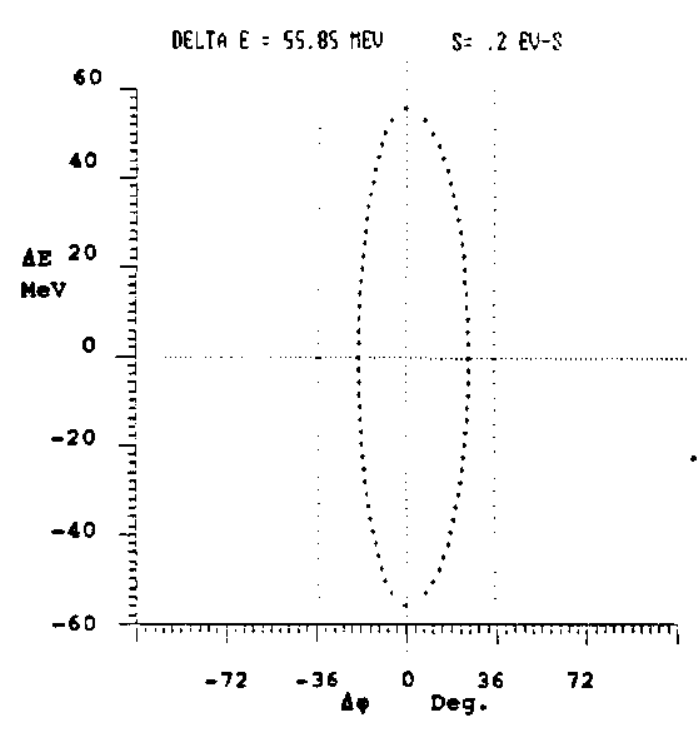

(a)

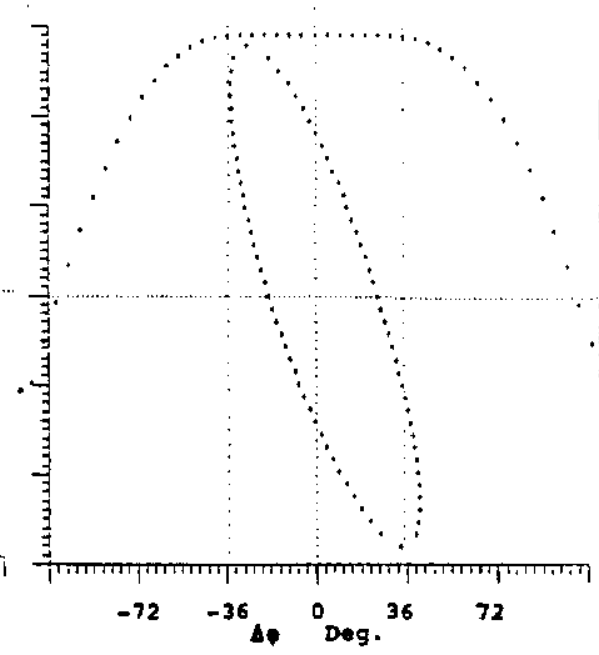

(b)

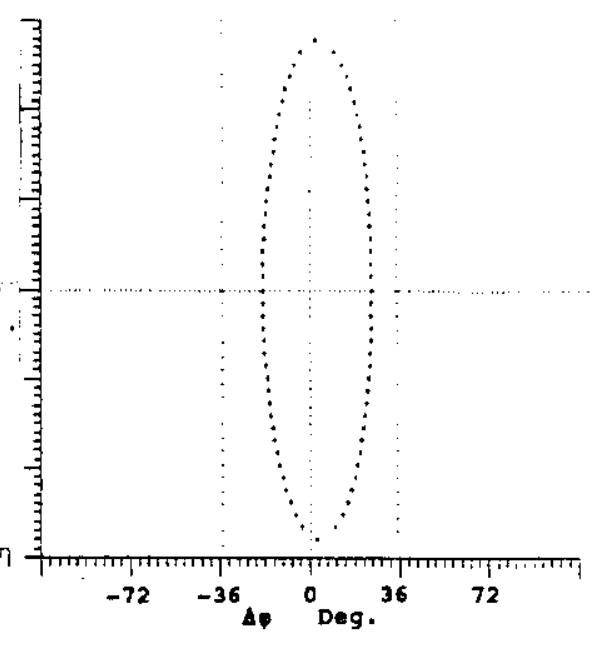

(c)

Figure 7. Phase space contours for 0.2 ev-s bunches as predicted by calculations for non-focussing transition crossing. (a) $6 \mathrm{~ms}$ prior to transition. (b) Transition. (c) $6 \mathrm{~ms}$ after transition.

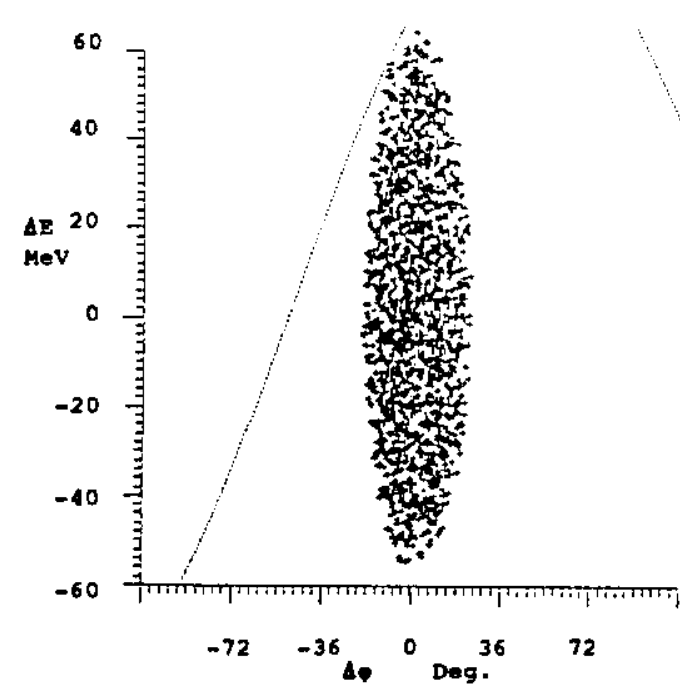

(a)

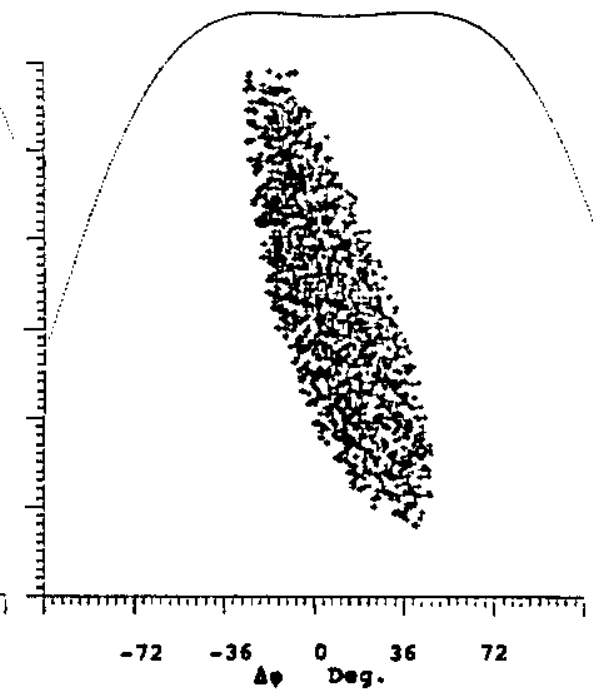

(b)

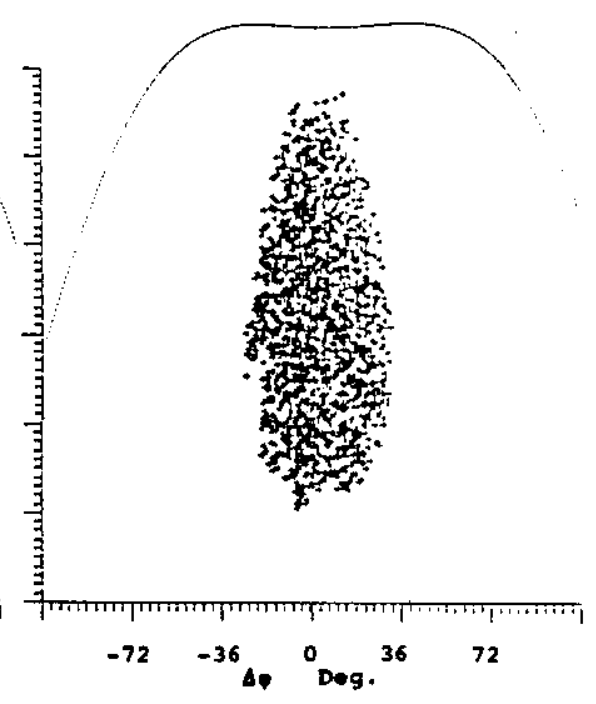

(c)

Figure 8. ESME simulation, $0.2 \mathrm{eV}-\mathrm{s}$ bunch containing $3 \times 10^{10}$ protons crossing transition in Fermilab Main Ring. $12 \mathrm{~ms}$ non-focussing period centered on transition time. 
distribution extent well. At transition space charge forces have broadened the distribution perceptably and the energy spread is reduced to $+50,-45 \mathrm{MeV}$. By the end if the non-focussing period, part $c$, the bunch has lengthened to $\$ 28.5$ degrees (full length $=3 \mathrm{~ns}$ ), the energy spread has reduced to $+46,-37 \mathrm{MeV}$ and some minor shape distortion is evident. All of the charge can now be captured within an accelerating bucket although a minor mismatch, leading to some emittance growth, is inevitable. This transition crossing is summarized in Fig.9 where the initial distribution at $15 \mathrm{GeV}$ is compared to the distribution in a post-transition accelerating bucket at $26 \mathrm{GeV}$. All of the initial charge has been recaptured and the longitudinal emittance has grown about 22 percent.

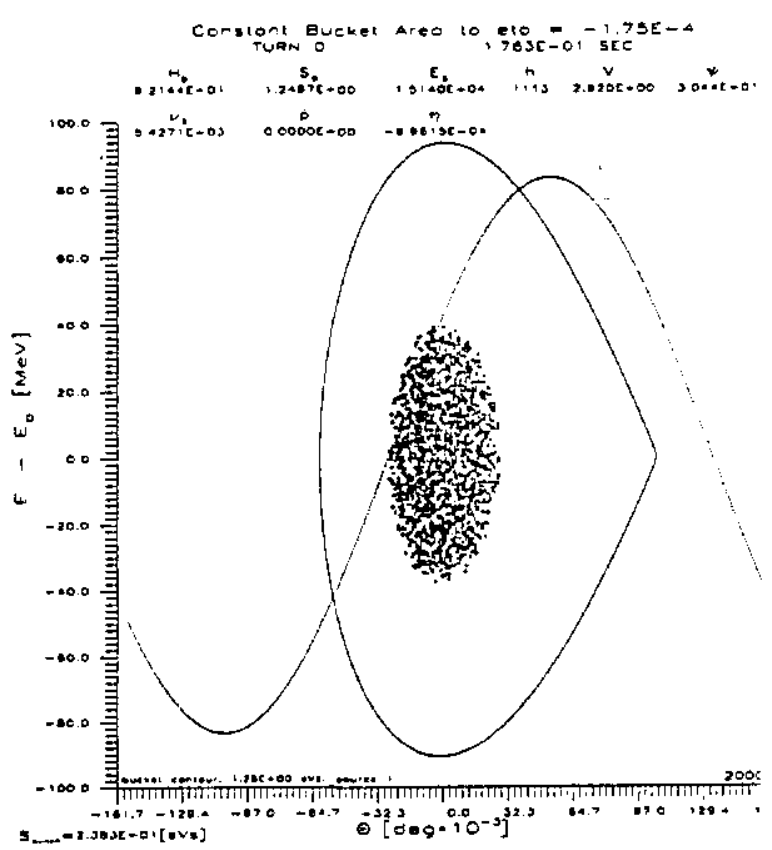

(a)

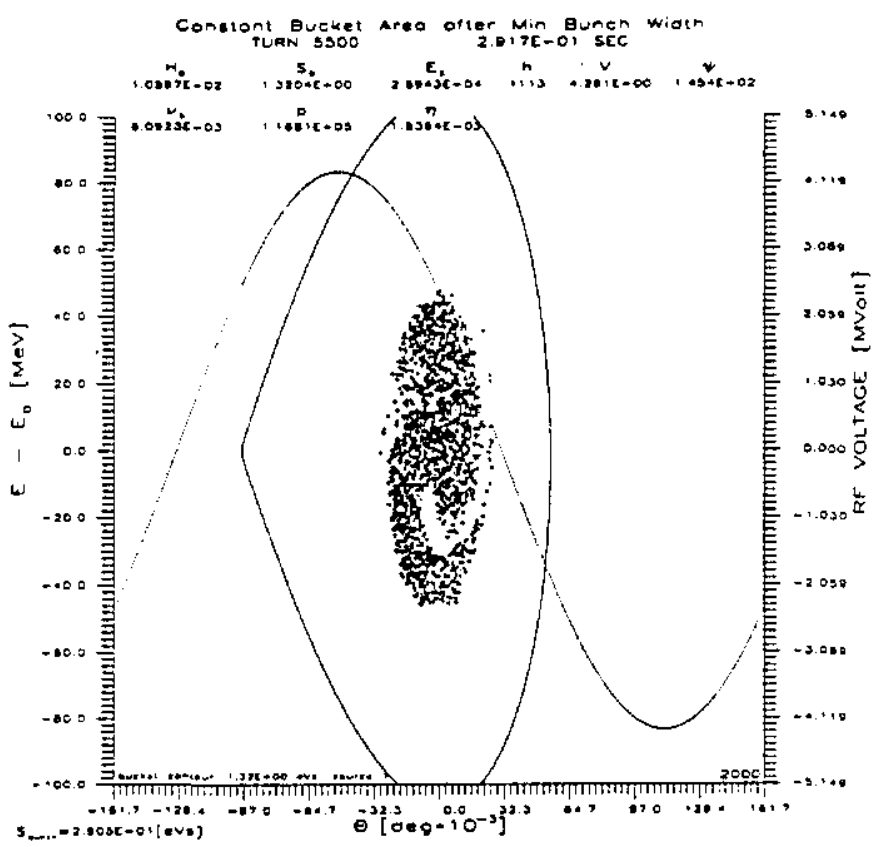

(b)

Figure 9. (a) $0.2 \mathrm{ev-s}$ distribution of $3 \times 10^{10}$ protons matched to a 1.25 ev-s accelerating bucket at $15 \mathrm{GeV}$. (b) The same bunch matched to a $1.32 \mathrm{eV}-\mathrm{s}$ accelerating bucket at $26 \mathrm{GeV}$, following non-focussing transition crossing. 
The second ESME simulation is that for a rather large bunch, $0.5 \mathrm{eV}-\mathrm{s}$ and $6 \times 10^{10}$ protons accelerated through transition in the proposed Fermilab Main Injector $\left(h=588, \gamma_{t}=20.4, \mathrm{z} / \mathrm{n}=5 \mathrm{Ohms}\right)$. In the example the acceleration rate is $152 \mathrm{GeV}$ per second and $\alpha_{1}$ is assumed to be 0.0033 . The momentum spread $\delta$ at the start of the non-focussing period is \pm 0.51 percent $(\approx \pm 100 \mathrm{MeV}$.$) . Using these parameters in Eqns.1$ and 8 , the total nonadiabatic time $t_{n}$ is $\pm 4.3 \mathrm{~ms}$. The optimum total bunch length, as determined from Eq.15, would be about 65 degrees. The shearing time used in the example is $4.15 \mathrm{~ms}$ and the total bunch length at the beginning of crossing is about 62 degrees. The non-focussing wave has been delayed 7 degrees to better match the extent of the sheared distribution. The distribution is shown at the beginning, at transition, and at the end of the shearing period in parts $a, b, a n d ~ c$ of Fig.10. Part $c$ shows the distribution captured in an accelerating bucket. The bucket is distorted toward lower momentum (closer to transition) and the charge distribution is distorted toward later time. Each of these effects is the result of the use of an abnormally large value for $\alpha_{1}$ in the simulation; a value between 0 and 0.001 would have been more realistic. Fig.10 d shows the distribution entirely captured in an accelerating bucket $14 \mathrm{~ms}$ after transition with only a few percent increase in longitudinal emittance. At this point the energy spread is not markedly different than it was at the beginning of the transition crossing period. 


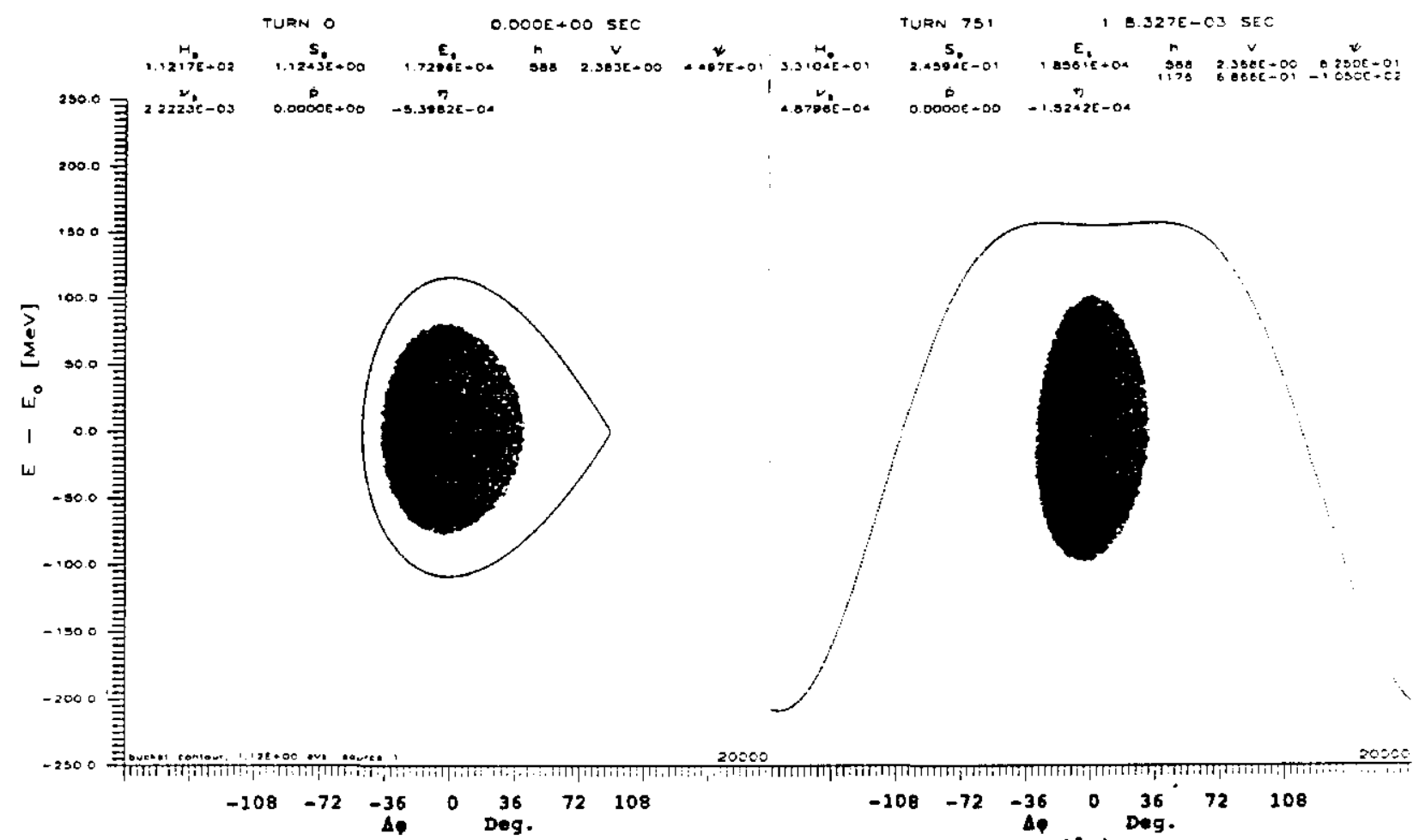
(a)

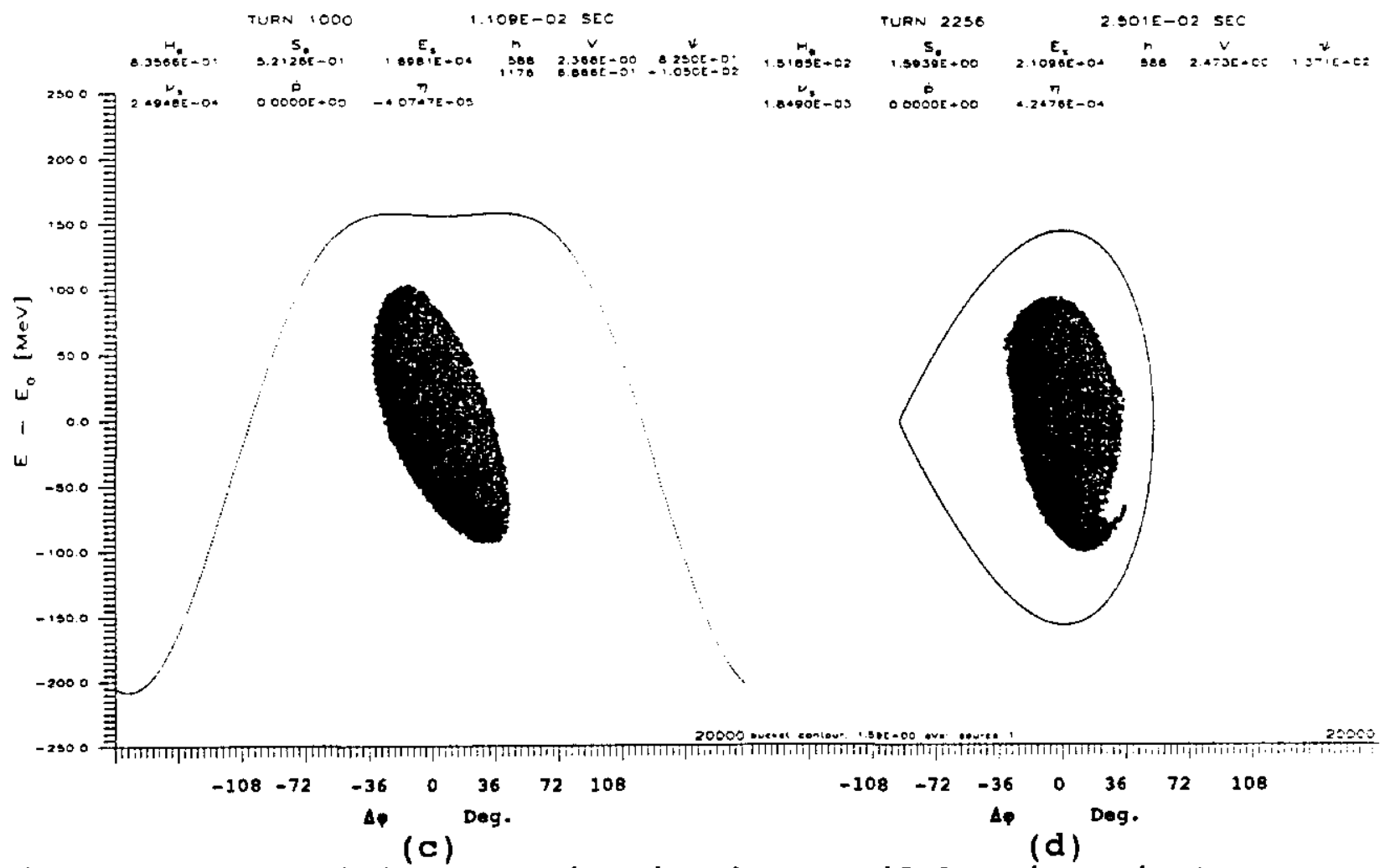

Figure 10. Transition crossing in the Fermilab Main Injector. 


\section{RF AMPLITUDE CONTROL; RADIAL POSITION CONTROL}

During normal acceleration the total rf amplitude and synchronous phase angle are programmed to provide the required acceleration voltage and bucket area. These programs can be generated from the magnetic guide field program $B(t)$, some knowledge of the beam longitudinal emittance, and some judgement regarding the desired bunch to bucket area ratio. The amplitude control program is not necessarily a high precision program, in part because one of the many rf stations in the system may malfunction slightly or possibly fail totally during acceleration and this should not be allowed to affect successful acceleration in a major way. Small discrepancies between the rf amplitude and that required to accelerate the beam on the reference orbit are corrected automatically by the radial position feedback system. That system detects movement of the beam charge center off of the reference orbit at one or more high dispersion points on the orbit and generates a radial position error signal. The error signal is added to the synchronous phase angle program so that the synchronous phase is changed slightly to provide the correct accelerating voltage. In so doing, the bucket area is also changed through the "moving bucket factor". If the rf amplitude is such that the required accelerating voltage can only be established by reducing the bucket area to a value smaller than the bunch longitudinal emittance then loss of some or all of the beam will occur.

These procedures for rf amplitude control and radial position control cannot function in the manner described above during the nonfocussing transition crossing period. Because the rf accelerating voltage is constant over a large phase range centered on the bunch position, small changes in the rf phase in response to a radial position error signal would not cause a correction in the acceleration rate and a large change would destroy the required phase relationship between the bunch position and the rf wave. Some form of feedback control of the beam position will have to be implemented during this period however. A 1 percent error in the accelerating voltage (about $18 \mathrm{kV}$ in the Fermilab Main Ring) would introduce an energy error of $10 \mathrm{MeV}$ in the entire distribution. An energy error of this size added to the already large beam energy spread could cause beam loss and would 
almost certainly exacerbate any bunch to bucket mismatch which occurs at the end of the non-focussing period. There is no assurance, of course, that open loop operation of the amplitude system would restrain voltage errors to the level of 1 percent. This situation will have to be corrected by switching to a feedback beam position control system in which the radial position error signal is added, is some form, to the rf amplitude control program signal during the non-focussing period. The stability dynamics (i.e. gain-phase relationship and transfer function stability) for this feedback system will have to be worked out.

In order to control the amplitude and phase of the second or third harmonic rf system during transition crossing, it will be necessary to implement additional feedback systems. A calibrated signal representing the amplitude of the harmonic if wave could be compared to a selected fraction of a similar signal representing the fundamental rf wave (the rf fan-back signal). The error signal so derived would then be added to an amplitude program signal delivered to the harmonic system. A similar feedback comparison system will have to be installed to compare the phase of the two rf waves.

In addition to the above described control systems the phase and amplitude of the harmonic rf system will have to be made insensitive to beam induced voltages both during operation and during the remainder of the acceleration cycle. This might be done by using standard amplitude and phase control as is done with other rf systems in the accelerator and switching in as substantial shunt impedance damping system at other times. Alternatively the system may be operated with a "local" rf feedback loop of sufficient loop gain to lower the output impedance to a satisfactory level. If this approach were taken the rf drive system could be left on during the entire accelerating cycle and the gap voltage programmed to zero at times other than during transition crossing. The local feedback system would then assume the role of gap impedance reduction by simply enforcing zero (or acceptably low) gap voltage except when needed. In any case the harmonic if system will have to be tunable over some frequency range, at least sufficient to cover the small frequency swing of the reference beam during transition crossing. 


\section{SUMMARY AND CONCLUSTONS}

In certain large dynamic range proton synchrotrons there is an energy at which the rotation frequency of particles is nearly independent of momentum within the momentum acceptance range. Near this "transition" energy particles cannot move azimuthally (with respect to the position of a set of reference particles) rapidly enough to execute complete phase oscillations about the reference phase. Consequently, those "off phase" particles are subjected rf accelerating voltage larger or smaller than necessary for a substantial period of time. This causes growth in the energy spread and longitudinal emittance of particle bunches in the machine and possible to particle loss.

A simple mechanism has been described whereby all of the particles in the machine are subjected to the correct accelerating voltage during the transition crossing period. This is accomplished through the addition of a second or third harmonic component of appropriate amplitude and phase to the accelerating $r f$ wave. The phase of the fundamental $r f$ wave is also adjusted to an optimum position.

It has been demonstrated through the use of large scale simulation that this technique is capable of transmitting high intensity bunches through transition with minimal emittance growth and no beam loss.

The examples given here have used a second harmonic component only. Use of the third harmonic is technically easier but the range of initial intensity and emittance is somewhat limited. Nevertheless, performance of the Fermilab Main Ring could be improved sufficiently using only a third harmonic component so that an early experimental test of the principle proposed here is expedient.

\section{ACKNOWLEDGMENT}

The author wishes to acknowledge the enthusiastic encouragement and assistance of James $A$. MacLachlan in the development of these ideas and the associated ESME simulations. Also thanks to $\mathrm{J}$. Wei of Brookhaven National Laboratory and S. Y. Lee of Univ. of Indiana for stimulating discussions regarding transition crossing in RHIC held on several occasions over the past several years. 


\section{REFERENCES}

1. E.D. Courant and H.S. Snyder, Theory of the Alternating-Gradient Synchrotron, ANNALS OF PHYSICS, $\underline{3}$ 1-48 (1958).

2. K.R. Symon and A.M. Sessler, Methods of Radio Frequency Acceleration in Fixed Field Accelerators with Applications to High Current and Intersecting Beam Accelerators, Proc. of the CERN Symposium on High Energy Accelerators and Pion Physics, 1,46, (1956).

3. J. Livingood, Principles of Cyclic Particle Accelerators, Ch. 6, D. Van Nostrand, New York, (1961).

4. A.A. Kolomensky and A.N. Lebedev, Theory of Cyclic Accelerators, pp190ff, North Holland, Amsterdam, (1966).

5. A. Sørenssen, Crossing the Phase Transition in Strong-Focusing Proton Synchrotrons, Particle Accelerators, 6 141-165, (1975).

6. A. Sørenssen, The Effect of Strong Longitudinal Space-Charge Forces at Transition Energy, Proc. 6th Internat. Conf. on HighEnergy Accelerators, Cambridge (USA), 474, (1957).

7. D. Möhl, Compensation of Space-Charge Effects at Transition by an Asymmetric Q Jump, CERN-ISR/300/GS/69-72

8. A. Hofmann, CERN 77-13. p139, (Geneva, 1977).

9. K. Takayama, Phase Dynamics Near Transition in the FNAL Main Ring, Fermilab FN-372, (1983).

10. Ng. K.Y., Some Estimation Concerning Transition Crossing of the Main Injector, Fermilab TM-1670, (1990).

11. J. Wei, Longitudinal Dynamics of the Non-Adiabatic Regime on Alternate Gradient Synchrotrons. PhD Dissertation, State Univ. N.Y, Stony Brook, (1989).

12. N.C. Christofilos, First proposed "duck-under" transition crossing. Private Communication, E.D. Courant.

13. J.A. MacLachlan, ESME: Longitudinal Phase Space Particle Tracking Program Documentation, Fermilab TM-1274, (1984).

14. S.Y. Lee and J.M. Wang, Microwave Instability Across the Transition Energy, IEEE Trans. Nucl. Sci. NS-32, No. 5. 2323, (1985).

15. C.E. Nielsen and A.M. Sessler, Longitudinal Space Charge Effects in Particle Accelerators, Rev. Sci. Instr., 30 No.2, 80, (1959).

16. K. Johnsen, Effects Non-linearities on the Phase Transition, Proc. of the CERN Symposiom on High Energy Accelerators and Pion Physics, $1,106,(1956)$. 
17. I. Kourbanis, J. Maclachlan, and K. Meisner, Measurement of the Momentum Dependance of Orbit Length in the (Fermilab) Main Ring, Accel. Expt. 172, (1991).

18. A. Bogacz and S. Peggs, Comments on the Behavior of $\alpha_{1}$ in Main Injector $\gamma_{t}$ Jump Schemes, Proc. of the Fermilab III Instabilities Workshop, 192, Fermilab, (1990).

19. F.T. Cole and P.L. Morton, Areas and Bunching Factors of Partially Filled Buckets, UCID 10130 AS/Theoretical/02, (1964). 